\title{
The Unique Dopamine/Ecdysteroid Receptor Modulates Ethanol-Induced Sedation in Drosophila
}

\author{
Emily Petruccelli, ${ }^{1} \mathbb{Q}^{Q} \mathrm{Li} \mathrm{Li}^{3}{ }^{3}$ Yi Rao, ${ }^{3}$ and $\mathbb{C}$ Toshihiro Kitamoto ${ }^{1,2}$ \\ ${ }^{1}$ Interdisciplinary Program in Genetics, Graduate College, ${ }^{2}$ Department of Anesthesia, Carver College of Medicine, University of Iowa, Iowa City, Iowa \\ 52242, and ${ }^{3}$ Peking University School of Life Sciences, Peking-Tsinghua Center for Life Sciences, Beijing 100871, China
}

Steroids profoundly influence behavioral responses to alcohol by activating canonical nuclear hormone receptors and exerting allosteric effects on ion channels. Accumulating evidence has demonstrated that steroids can also trigger biological effects by directly binding G-protein-coupled receptors (GPCRs), yet physiological roles of such unconventional steroid signaling in controlling alcohol-induced behaviors remain unclear. The dopamine/ecdysteroid receptor (DopEcR) is a GPCR that mediates nongenomic actions of ecdysteroids, the major steroid hormones in insects. Here, we report that Drosophila DopEcR plays a critical role in ethanol-induced sedation. DopEcR mutants took longer than control flies to become sedated during exposure to ethanol, despite having normal ethanol absorption or metabolism. RNAi-mediated knockdown of DopEcR expression revealed that this receptor is necessary after eclosion, and is required in particular neuronal subsets, including cholinergic and peptidergic neurons, to mediate this behavior. Additionally, flies ubiquitously overexpressing DopEcR cDNA had a tendency to become sedated quickly upon ethanol exposure. These results indicate that neuronal subset-specific expression of DopEcR in adults is required for normal sedation upon exposure to ethanol. We also obtained evidence indicating that DopEcR may promote ethanol sedation by suppressing epidermal growth factor receptor/extracellular signal-regulated kinase signaling. Last, genetic and pharmacological analyses suggested that in adult flies ecdysone may serve as an inverse agonist of DopEcR and suppress the sedation-promoting activity of DopEcR in the context of ethanol exposure. Our findings provide the first evidence for the involvement of nongenomic G-protein-coupled steroid receptors in the response to alcohol, and shed new light on the potential roles of steroids in alcohol-use disorders.

Key words: alcohol; dopamine; Drosophila; EGFR; GPCR; steroid

\section{Significance Statement}

Alcohol abuse is an alarming personal and societal burden. The improvement of prevention and treatment strategies for alcoholuse disorders requires a better understanding of their biological basis. Steroid hormones profoundly affect alcohol-induced behaviors, but the contribution of their unconventional, nongenomic actions during these responses has not yet been elucidated. We found that Drosophila DopEcR, a unique G-protein-coupled receptor (GPCR) with dual specificity for dopamine and steroids, mediates noncanonical steroid actions to promote ethanol-induced sedation. Because steroid signaling and the behavioral response to alcohol are evolutionarily well conserved, our findings suggest that analogous mammalian receptors likely play important roles in alcohol-use disorders. Our work provides a foundation for further characterizing the function and mechanisms of action of nonclassical steroid GPCR signaling.

\section{Introduction}

The fruit fly Drosophila melanogaster expresses a unique G-protein-coupled receptor (GPCR) called the dopamine/ecdys- teroid receptor (DopEcR), which can be activated by both the major insect hormone ecdysone and the catecholamine dopa-

technical assistance, members of the Kitamoto laboratory for fruitful discussions, and the Bloomington Stock Center and VDRC for fly stocks.

The authors declare no competing financial interests.

Correspondence should be addressed to Toshihiro Kitamoto, Department of Anesthesia, Carver College of Medicine, University of lowa, lowa City, 1-316 Bowen Science Building, 51 Newton Road, lowa City, IA 52242. E-mail: toshi-kitamoto@uiowa.edu.

E. Petruccelli's present address: Department of Neuroscience, Brown University, Providence, RI 02912.

DOI:10.1523/JNEUROSCI.3774-15.2016

Copyright $\odot 2016$ the authors $\quad 0270-6474 / 16 / 364647-11 \$ 15.00 / 0$ 
mine (Srivastava et al., 2005). In heterologous cell culture systems, DopEcR induces rapid mitogen-activated protein kinase/ extracellular signal-related kinase (MAPK/ERK) signaling in response to ecdysteroids, and cAMP/PI3K signaling in response to dopamine. Although DopEcR is insect specific, it is similar in sequence to vertebrate $\beta 2$-adrenergic receptors and similar in function to the mammalian G-protein-coupled estrogen receptor GPER1 (formerly known as GPR30; Srivastava et al., 2005; Evans et al., 2014). Recent studies have revealed various roles for DopEcR in vivo. For instance, DopEcR can act as a dopamine receptor in primary gustatory neurons to control the proboscis extension reflex (Inagaki et al., 2012) and as a nongenomic ecdysone receptor in mushroom body neurons to regulate experience-dependent courtship suppression (Ishimoto et al., 2013). In Agrotis ipsilon moths, DopEcR requires both dopamine and ecdysone as ligands to modulate the perception of sex pheromones (Abrieux et al., 2013, 2014).

Intriguingly, several signaling components associated with DopEcR — steroids, dopamine, cAMP, and ERK— - have been implicated in evolutionarily conserved behavioral responses to alcohol (Moore et al., 1998; Bainton et al., 2000; Koob, 2008; Corl et al., 2009; Kong et al., 2010). Like in mammals, flies acutely exposed to a constant flow of ethanol vapor will increase locomotion, lose postural control, and eventually become sedated (Wolf et al., 2002). Thus, studies using Drosophila as a genetically tractable model system have facilitated the discovery and analysis of various genes linked to alcohol-use disorders (AUDs) (Kaun et al., 2012; Devineni and Heberlein, 2013). Although we lack a comprehensive understanding of the complex physiology underlying the pathological conditions of AUDs, the importance of steroid hormones in modulating behavioral responses to alcohol has long been appreciated (Devaud et al., 2006). Steroids classically exert their biological effects by binding cognate nuclear receptors and altering gene transcription. However, they are also known to elicit rapid cellular actions by directly activating GPCRs (Lösel and Wehling, 2003; Olde and Leeb-Lundberg, 2009), and the consequences of such nongenomic actions of steroids in the nervous system, particularly in the context of alcohol-related behaviors, remain unknown.

Here, we show that Drosophila DopEcR is necessary for normal levels of ethanol-induced sedation in flies. Our data indicate that DopEcR suppresses epidermal growth factor receptor (EGFR)/ERK signaling to promote ethanol-induced sedation, and that ecdysone can negatively regulate this effect during ethanol exposure. These findings contribute to our knowledge of GPCR-mediated, nongenomic actions of steroids and provide novel insight into the neurobiological mechanisms underlying behavioral responses to alcohol.

\section{Materials and Methods}

Fly stocks. Flies were raised on standard cornmeal/agar food at $25^{\circ} \mathrm{C}$ in $65 \%$ humidity under a $12 \mathrm{~h}$ light/dark cycle. For all experiments, newly eclosed males were collected over a $2 \mathrm{~d}$ period, housed 10/vial, and aged 3-5 d. Two DopEcR mutant alleles were used in this study. A hypomorphic allele DopEcR ${ }^{P B 1}$ (also known as DopEcR ${ }^{c 02142}$; Ishimoto et al., 2013) that carries an intronic piggyBac transposon insertion was obtained from the Bloomington Stock Center. The presumptive null allele $D o p E c R^{G A L 4}$ was generated by replacing part of the first coding exon of DopEcR (133 bp from 4370598 to 4370730) with GAL4 and GMR-white. Genetargeting experiments were performed as describe previously (Huang et al., 2008). DopEcR ${ }^{P B 1}$ and $D o p E c R^{G A L 4}$ mutants were backcrossed six generations to the $w^{1118}$ strain obtained from the Vienna Drosophila RNAi Center (VDRC). UAS-myrGFP-2A-dsRednls (Daniels et al., 2014) was shared by Dr. Barry Ganetzky (University of Wisconsin, Madison).
UAS-DopEcR-RNAi (KK111211) and UAS-Egfr-RNAi (KK107130) were obtained from the VDRC phiC31 RNAi library. UAS-DopEcR-cDNA was created previously in our laboratory (Ishimoto et al., 2013). The GAL4 lines, UAS-GFP, DTS-3 (also known as $m l d^{D T S-3}$ ), and $D o p R^{P L 00420}$ were obtained from the Bloomington Stock Center, and the $D A T^{f m n}$ flies were a gift from Dr. Kazuhiko Kume (Nagoya City University, Nagoya, Japan).

Immunostaining and confocal microscopy. Immunostaining was performed essentially as described in Ishimoto et al. (2013). Briefly, adult male brains were dissected, fixed, blocked, and stained with either mouse anti-Elav IgG (1:500) or mouse anti-Repo IgG (1:200; Developmental Studies Hybridoma Bank, University of Iowa), followed by goat Alexa Fluor 647-conjugated anti-mouse IgG (1:500; Invitrogen). A confocal microscope (Olympus FV1000 or Zeiss 510) was used to collect $z$-sections of the adult brain, expressing fluorescent reporters (GFP or RFP) or stained with antibodies, at 1 or $2 \mu \mathrm{m}$ intervals with a $20 \times$ objective lens. ImageJ was used to pseudocolor and create maximum intensity $z$-projections. For colocalization analysis of two fluorescent signals, the default settings of ImageJ plug-in Colocalization were applied to stacks of $10-\mu \mathrm{m}$-deep horizontal sections.

Behavioral assays. Sensitivity to ethanol was assessed using similar methods described in previous studies (Chen et al., 2008; Cavaliere et al., 2012). Approximately 10 3-5-d-old flies were transferred from regular vials containing food to empty polystyrene vials $(22.5 \times 90 \mathrm{~mm})$ with a cotton-ball stopper. Following $5 \mathrm{~min}$ for acclimation, $0.5 \mathrm{ml}$ of $50 \%$ ethanol was applied to the center of the cotton ball, and the vials were quickly sealed with Parafilm and placed on a white background under constant light. Without mechanical stimulation, flies were scored every 5 min for "loss of postural control" (on vial bottom) and "sedation" (akinesia, i.e., on vial bottom, not standing in a normal posture, and lack of movement other than small twitches). The percentage of flies scored at each time point was calculated, and the time for $50 \%$ of flies to be scored-loss of postural control [time for $50 \%$ of flies to lose postural control (LT50)] and sedation [the time for $50 \%$ of flies to become sedated (ST50)] was determined by linear interpolation. Graphs represent the average and SEM across vials ( $n=$ the number of vials tested). All ethanol-sensitivity assays, including those using temperature-shift paradigms, were performed at $25^{\circ} \mathrm{C}$ in $65 \%$ humidity between zeitgeber time (ZT) 0 and ZT6.

Ethanol absorption and metabolism assay. Analysis of internal ethanol levels was performed as described in previous studies (Moore et al., 1998; Wolf et al., 2002; Corl et al., 2009). Approximately 30 flies were subjected to the ethanol-sensitivity assay for 0,15 , or $30 \mathrm{~min}$ (absorption), or for $30 \mathrm{~min}$ and were then allowed to recover in the presence of food for 30, 60, or $120 \mathrm{~min}$ (metabolism). Flies were then immediately transferred to empty polystyrene vials, frozen at $-80^{\circ} \mathrm{C}$, homogenized in $300 \mu \mathrm{l}$ of $50 \mathrm{~mm}$ Tris $\mathrm{HCl}, \mathrm{pH} 7.5$, and centrifuged for $20 \mathrm{~min}$ at $4^{\circ} \mathrm{C}$. Homogenate $(2 \mu \mathrm{l})$ was added to alcohol reagent $(200 \mu \mathrm{l})$ from an ethanol assay kit (catalog \#229-29, Genzyme Diagnostics Sekisui). After $20 \mathrm{~min}$ incubation at room temperature, $\mathrm{OD}_{340}$ readings were taken using plate spectrophotometry. Readings from technical replicates were averaged, and ethanol concentrations were derived from standard curves. Experiments were performed $\geq 4$ times independently. The water content of a single male fly was assumed to be $0.6 \mu \mathrm{l}$, in accordance with the findings of Devineni and Heberlein (2012).

Western blot analysis. Western blot analysis was performed essentially as described by Harlow and Lane (1988). Approximately $303-5$-d-old flies were transferred to empty polystyrene vials, frozen at $-80^{\circ} \mathrm{C}$, and briefly vortexed to dissociate heads. Heads were then homogenized in $2 \times$ Laemelli's loading buffer ( $5 \mu \mathrm{l} / \mathrm{head})$, and the homogenates were centrifuged at 13,200 rpm for $10 \mathrm{~min}$. Supernatant [ $12 \mu \mathrm{l}$ for phosphorylated ERK (p-ERK) and total ERK; $4 \mu$ l for tubulin] was run on 10\% TGX polyacrylamide gels (Bio-Rad). Protein bands were transferred to PVDF Immobilon membranes (Millipore) using a semidry system, and probed with mouse anti-diphospho-MAPK (1:2000; Sigma M8159), rabbit antiMAPK (1:2000; Sigma M5670), or mouse anti-tubulin (1:10,000; Sigma T9026) antibodies (Sigma-Aldrich). Antigen-antibody complexes were then detected using appropriate goat anti-IgG antibodies conjugated 
A

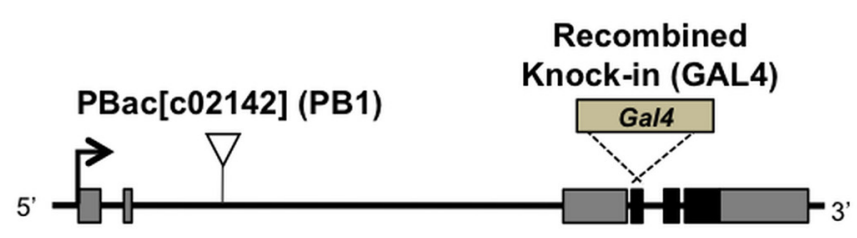

\section{DopEcR}

3L: $4,380,387 . .4,367,649$

C

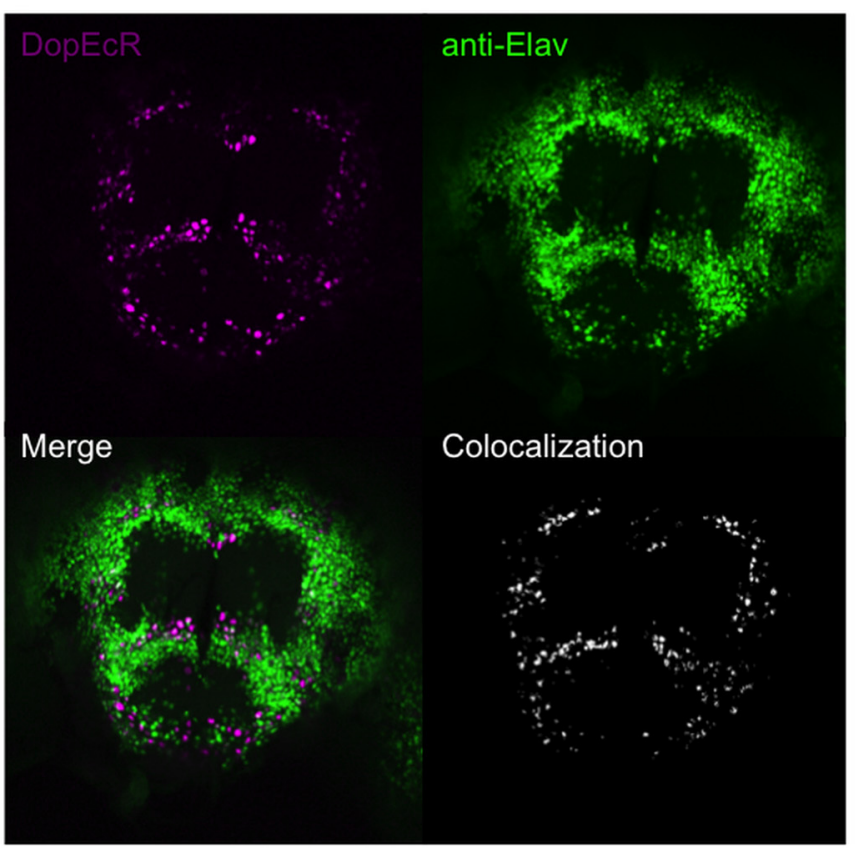

B

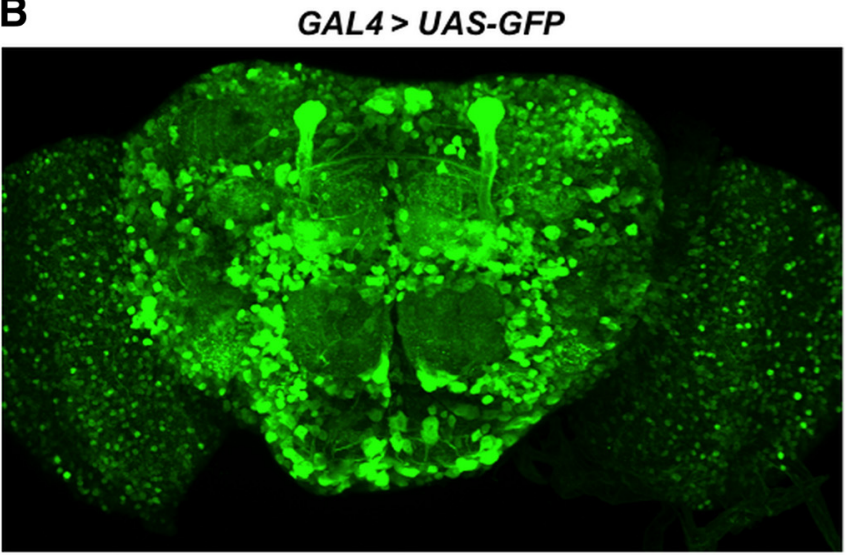

D

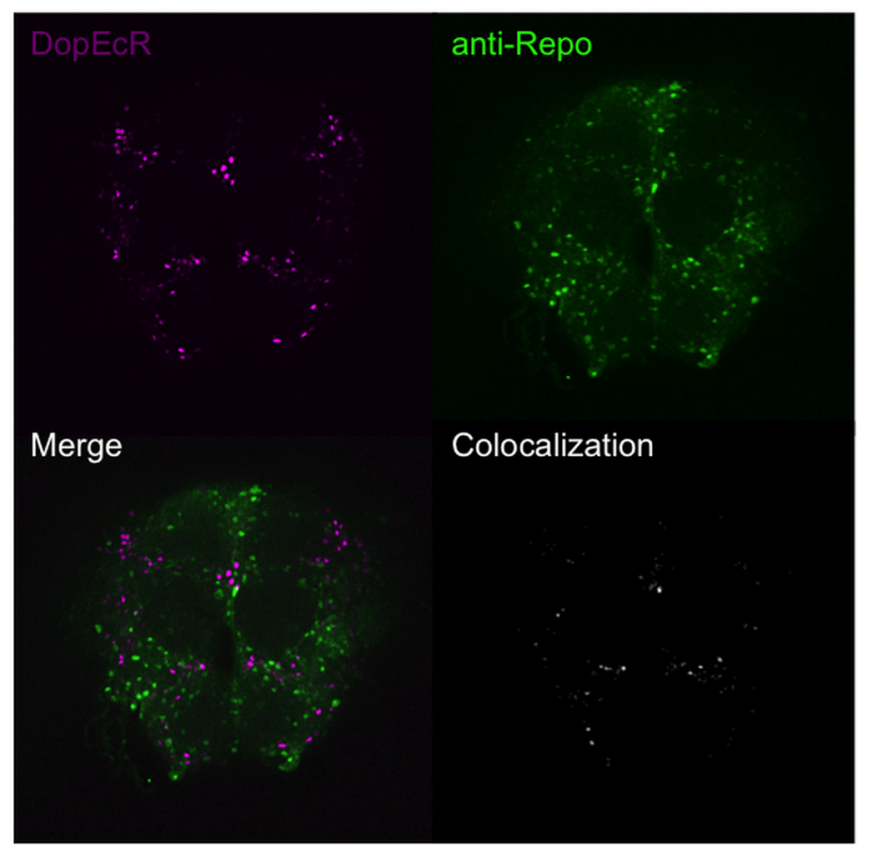

Figure 1. DopECR mutants and the endogenous DopEcR expression pattern in the brain deduced using a GAL4 knock-in allele, DopECR ${ }^{G A L 4}$. A, DopECR genomic locus illustrating the piggyBac transposon insertion mutant $D o p E C R^{c 02142}(P B 1)$ and GAL4 knock-in mutant $D O p E C R^{G A L 4}(G A L 4)$. An arrow, gray boxes, and black boxes depict the transcription start site, exons, and coding region, respectively, of the DopEcR gene. $\boldsymbol{B}$, Anterior view of the adult brain displaying GFP reporter expression driven by GAL4 activity associated with $D o p E C R^{G A L 4}$. C, D, Double labeling of the adult brain (DopECR ${ }^{\text {GAL }} /$ UAS-myrGFP-2A-dsRedn/s) for nuclear RFP reporter for DopEcR (magenta) and neuronal marker Elav ( $\boldsymbol{C}$, green) or glial marker Repo (D, green). Colabeling (white) is visualized using ImageJ plug-in Colocalization.

with HRP (1:10,000; Santa Cruz Biotechnology). Membranes were exposed to the Western Lightning ECL-Plus reagent (PerkinElmer Life and Analytical Sciences), and signals were developed on Kodak-Biomax x-ray film (Eastman Kodak). ImageJ software was used for densitometric analysis (Schneider et al., 2012).

Feeding of 20-hydroxyecdysone. A $50 \mathrm{~mm}$ stock solution of 20-hydroxyecdysone (20E; Sigma-Aldrich) was prepared in $70 \%$ ethanol and added to reheated food. For vehicle controls the same amount of $70 \%$ ethanol was added. Three-to-five-day-old adult male flies, raised 10/vial, were transferred to vials with food containing $0.01 \mathrm{~mm} 20 \mathrm{E}$ or vehicle for $24 \mathrm{~h}$ before being tested in the ethanol-sensitivity assay.

Statistics. SigmaPlot (Systat Software) was used to determine statistical significance for ethanol-induced behaviors and Western blot results. Behavior over time was analyzed by two-way repeated-measures ANOVA on ranks, followed by Holm-Sidak multiple-comparisons procedures. LT50 and ST50 data were assessed using ANOVA on ranks, Dunn's, or Mann-Whitney rank-sum tests. The $\chi^{2}$ test was used to assess survival of sedated flies following $90 \mathrm{~min}$ exposure to ethanol. All graphs display data averages, with error bars representing SEM.

\section{Results}

A GAL4 knock-in allele indicates widespread neuronal expression of DopEcR in the adult brain

To examine the potential role of DopEcR in behavioral responses to alcohol, we used hypomorphic DopEcR $R^{P B 1}$ and null $D o p E c R^{G A L 4}$ mutants. The DopEcR ${ }^{P B 1}$ allele contains an intronic piggyBac transposon insertion that significantly reduces levels of the DopEcR transcript (Ishimoto et al., 2013), whereas the newly generated DopEcR ${ }^{G A L 4}$ knock-in mutant has an in-frame GAL4 construct in which part of the first coding exon is replaced, rendering it a null allele (Fig. $1 A$ ). Both $D o p E c R^{P B 1}$ and DopEcR $R^{G A L 4}$ homozygotes were viable, and displayed grossly normal development and morphology. DopEcR expression has previously been observed in the developing fly nervous system using in situ hybridization (Srivastava et al., 2005), and suggested in the adult nervous system through reporter gene expression driven by the putative DopEcR enhancer (Ishimoto et al., 2013). Here, we de- 
A

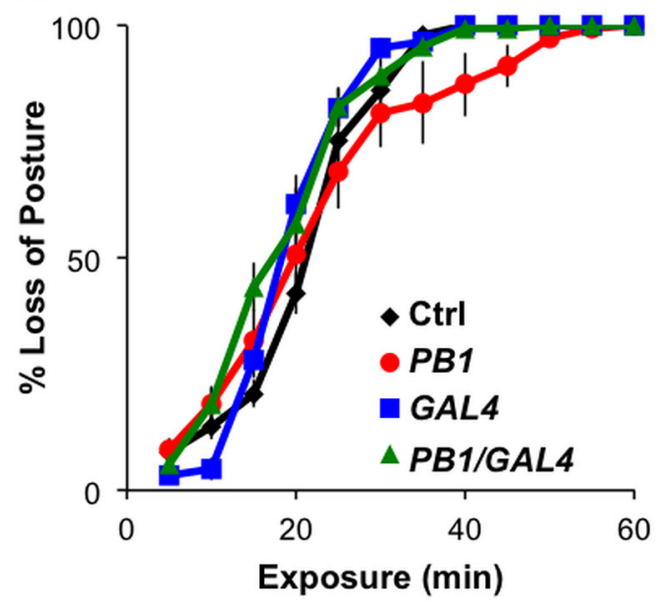

C

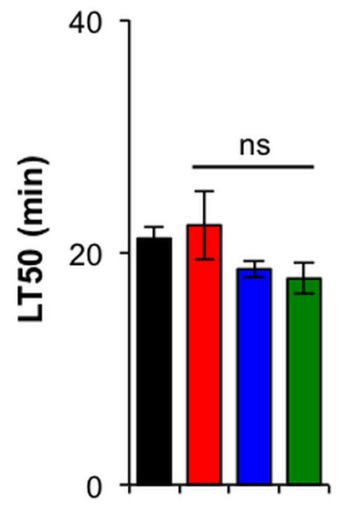

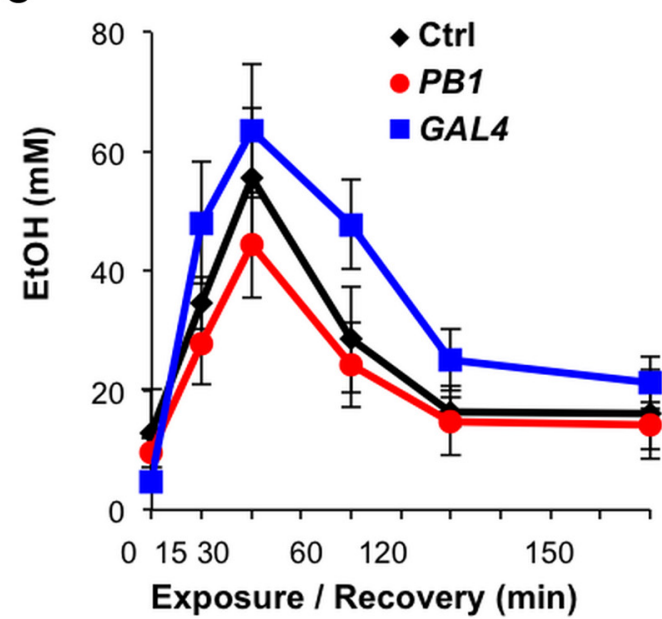

B
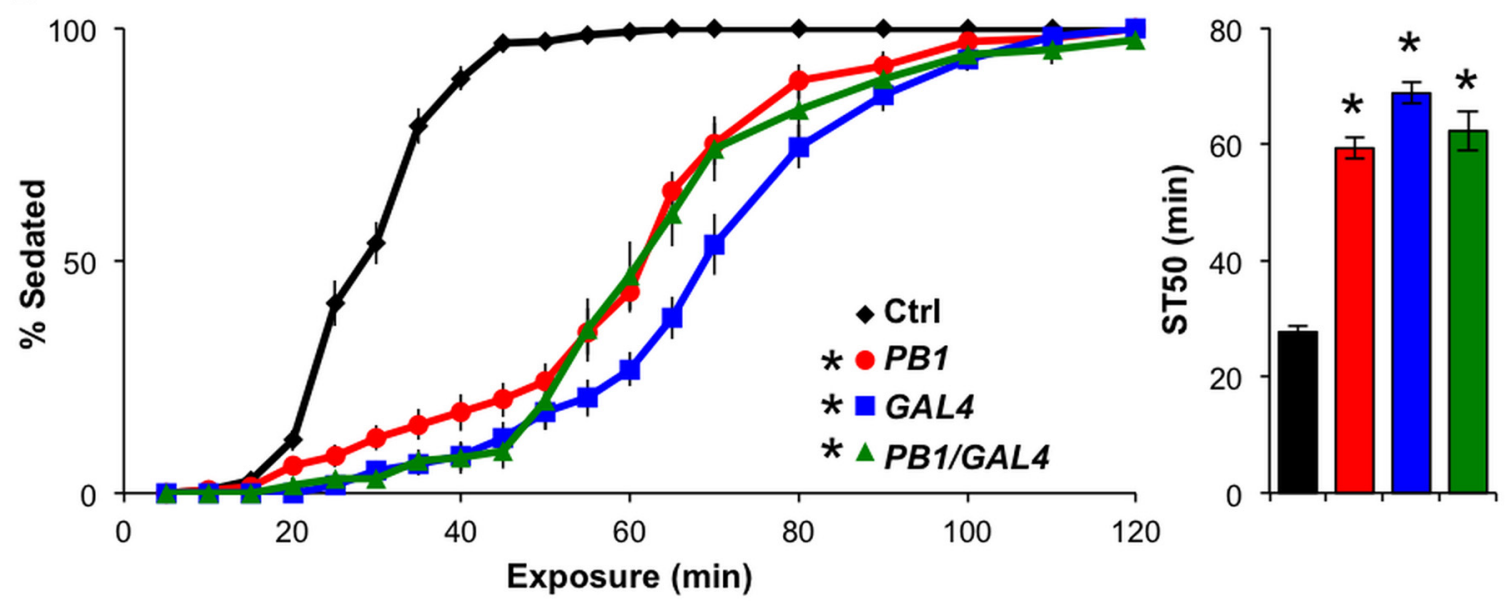

Figure 2. DopEcR mutants show increased resistance to ethanol-induced sedation. $\boldsymbol{A}, \boldsymbol{B}$, Behavioral responses of $D$ op $E c R$ mutants to ethanol vapor. Left, Percentage of flies exhibiting loss of posture $(\boldsymbol{A})$ or sedation $(\boldsymbol{B})$ as a function of ethanol exposure. Right, LT50 $(\boldsymbol{A})$ or ST50 $(\boldsymbol{B})$ as calculated by linear interpolation. Empty vials containing $8-10$ control $\left(C\right.$ trl; $\left.w^{1118}, n=15\right), P B 1$

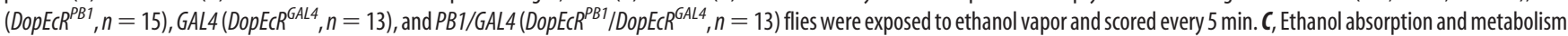
among genotypes ( $n=4$, each with $\sim 30$ flies). All figures display averages with SEM, with two-way repeated-measures ANOVA on ranks, Holm-Sidak ( $\boldsymbol{A}$ and $\boldsymbol{B}$, left; $\boldsymbol{C}$ ), and ANOVA on ranks, Dunn's ( $\boldsymbol{A}$ and $\boldsymbol{B}$, right) versus control, ${ }^{*} p<0.05$.

duced the endogenous DopEcR expression in the adult brain using GAL4 activity associated with the knock-in $D o p E c R^{G A L 4}$ allele. $D o p E c R^{G A L 4}$-driven GFP reporter expression was widely observed throughout the adult brain in UAS-GFP/+;DopEcR $R^{G A L 4} /+$ flies (Fig. 1B). Consistent with our previous findings (Ishimoto et al., 2013), the prominent neuropil structures, the mushroom bodies, were also GFP-positive (Fig. 1B). Next, using a nuclear localized RFP reporter (Daniels et al., 2014) for DopEcR (DopEcR ${ }^{G A L 4} / U A S-m y r G F P-2 A-$ $d s R e d n l s)$ in combination with antibodies against neuronal marker Elav (Fig. 1C) or glial marker Repo (Fig. 1D), we found that $D o p E c R^{G A L 4}$ expression was primarily observed in neurons but not glial cells.

\section{DopEcR mutants are resistant to ethanol-induced sedation}

To assess ethanol sensitivity, we subjected control and DopEcR mutant flies to ethanol vapor in an empty vial and observed their behavioral responses every $5 \mathrm{~min}$ for $2 \mathrm{~h}$. Upon exposure, flies gradually lost postural control, falling from the vial sides, and were increasingly seen on the vial bottom. Thus, the percentage of flies on the vial bottom was used as an indicator of ethanol-induced loss of postural control. Flies homozygous for $D o p E c R^{P B 1}$ or $D o p E c R^{G A L 4}$, and flies trans-heterozygous for the two alleles (DopEcR $\left.R^{P B 1} / D o p E c R^{G A L 4}\right)$ lost their posture around the same time as controls (Fig. 2A). LT50 values were $\sim 20 \mathrm{~min}$ for all genotypes. Moreover, $\sim 20 \mathrm{~min}$ into exposure, the control flies began to fail in all motor activity, other than minor twitching, and were no longer standing; these flies were considered to be sedated (Fig. $2 B$ ). Within $\sim 50$ min of exposure, nearly all of the control flies were sedated. In contrast, $D o p E c R$ mutants continued to move in an uncoordinated manner, typically while on their backs, as if trying to regain their posture, long after most control flies were sedated. It took nearly $2 \mathrm{~h}$ for all DopEcR mutants to become sedated. ST50 values were $\sim 30 \mathrm{~min}$ for control flies and $60 \mathrm{~min}$ for the DopEcR mutants (Fig. 2B). Heterozygous DopEcR $R^{P B 1}$ or $D o p E c R^{G A L 4}$ flies also showed significant resistance to sedation (ST50: $41 \pm 3 \min , n=10$ or $43 \pm 4 \min , n=14$, respectively) compared with controls, albeit to a lesser extent than homozygous mutants. Virgin $D o p E c R^{P B 1}$ or DopEcR ${ }^{G A L 4}$ females also exhibited resistance to ethanol-induced sedation (ST50: $75 \pm 3 \mathrm{~min}, n=8$ or $65 \pm 3 \mathrm{~min}, n=13$, respectively) compared with virgin female controls (ST50: $33 \pm 2 \mathrm{~min}, n=13$ ). 
We additionally addressed whether the absorption or metabolism of ethanol was abnormal in DopEcR mutants, measuring internal ethanol levels at various time points using an ethanol assay kit. Neither slow absorption nor fast metabolism of ethanol could explain the resistance to sedation in the mutants (Fig. 2C), suggesting that DopEcR modifies the behavioral response to alcohol by means other than influencing absorption or metabolism. Since DopEcR is expressed in the nervous system (Graveley et al., 2011), primarily in neurons (Fig. 1C), we hypothesized that this receptor plays a neurobiological role in promoting sedation during naive ethanol exposure.

\section{DopEcR mutants are more susceptible to death after ethanol sedation}

While performing the ethanol sensitivity assays, we observed that, unlike controls, a large proportion of sedated DopEcR mutants displayed a "wings up" phenotype commonly seen upon death. To determine whether DopEcR mutants are more likely than their wild-type counterparts to die following sedation, control and mutant flies were exposed to ethanol for 90 min and then allowed to recover on food for $24 \mathrm{~h}$. As compared with sedated control flies, significantly fewer sedated DopEcR mutants survived intoxication: $41 \% w^{1118}, n=220$; $4 \% D_{o p E c R^{P B 1}}, n=230 ; 25 \%$ DopEcR ${ }^{G A L 4}, n=177 ; X^{2}=$ $87.079, \mathrm{df}=2, p<0.001$. These findings suggest that, despite the fact that DopEcR mutants were more resistant to ethanol sedation than control flies, once they were sedated, they were less likely to survive. It is possible that the majority of DopEcR mutants never truly become sedated during ethanol exposure, but rather die when movement ceases.

\section{DopEcR expression is inversely correlated with resistance to ethanol-induced sedation}

To further validate a role for DopEcR in modulating ethanolinduced sedation, we manipulated the expression of DopEcR using the GAL4/UAS binary expression system (Brand and Perrimon, 1993). Ubiquitous knockdown of DopEcR with $d a-$ GAL4 and UAS-DopEcR-RNAi (for knockdown efficiency, see Ishimoto et al., 2013) led to significant resistance to ethanolinduced sedation similar to that observed in DopEcR mutants (Fig. 3A). This RNAi-induced phenotype was lessened upon simultaneous expression of a DopEcR cDNA transgene (ST50: $36 \pm 3.5 \mathrm{~min}, n=5$ ), suggesting that it was not an off-target effect of the RNAi. Furthermore, ubiquitous overexpression of $D o p E c R$ cDNA in a DopEcR wild-type background tended to reduce the time required for sedation, although the difference in values between experimental flies and UAS controls did not reach statistical significance (Fig. $3 B$ ). These results reveal an inverse relationship between levels of DopEcR expression and resistance to ethanol-induced sedation, further supporting the notion that DopEcR promotes sedation during exposure to ethanol.

\section{$D o p E c R$ is required in adulthood to promote ethanol-induced sedation}

Next, we asked when during development DopEcR expression is necessary for modulating the response to ethanol in adulthood. To this end, we used the temporal and regional gene expression targeting (TARGET) method (McGuire et al., 2004), which allowed for temporal control of DopEcR-RNAi expression via the temperature-sensitive GAL4 inhibitor, GAL $80^{\text {ts }}$. When flies were raised at $18^{\circ} \mathrm{C}$ throughout development and adulthood, the experimental genotype (UAS-DopEcR-RNAi/+;tub-GAL80 ${ }^{\text {ts }}$ / da-GAL4) did not exhibit resistance to ethanol sedation compared with either of its controls (UAS-DopEcR-RNAi/+;tub$G A L 80^{t s} /+$ and $+/+$; $+/ d a-G A L 4$; Fig. 3C). However, when raised continuously at $29^{\circ} \mathrm{C}$, the experimental flies showed greater ST50 values and exhibited significant resistance to sedation (Fig. 3D). The results of these constant-temperature paradigms assured us that the TARGET system was working as expected. Next, we chose to restrict DopEcR knockdown to before or after eclosion since $D o p E c R$ expression has been detected very early in development and throughout life (Srivastava et al., 2005; Graveley et al., 2011). When flies were reared at $29^{\circ} \mathrm{C}$ during the embryonic, larval, and pupal stages, and transferred to $18^{\circ} \mathrm{C}$ just after eclosion, experimental flies did not show resistance (Fig. $3 E$ ). However, when flies were reared at $18^{\circ} \mathrm{C}$ during development and then transferred to $29^{\circ} \mathrm{C}$ just after eclosion, the experimental group exhibited significant resistance relative to controls (Fig. 3F). These data suggest that posteclosion expression of DopEcR is necessary for proper ethanol sedation in adults.

\section{Knockdown of DopEcR in neurons enhances resistance to ethanol-induced sedation}

To determine the possible sites of DopEcR action for promoting ethanol-induced sedation, we examined the sedation response of flies expressing DopEcR-RNAi in a variety of cell populations, using cell type-specific GAL4 drivers. Significant increases in resistance to sedation were observed when DopEcR-RNA $i$ was expressed using a pan-neuronal driver, elav-GAL4, indicating that neuronal expression of $D o p E c R$ is required for normal ethanol sedation (Fig. 4A). Among GAL4 lines that drive expression in specific subsets of neurons, those driving cholinergic ( $\mathrm{Cha}$ GAL4) and pan-peptidergic (c929-GAL4) expression of DopEcR$R N A i$ increased resistance to ethanol sedation compared with both control genotypes (Fig. $4 B, C$ ). Knockdown of DopEcR with several other GAL4 lines, including mushroom body (c772GAL4), dopaminergic (TH-GAL4), and insulin-like peptide (dilp2-GAL4) drivers, had little effect on ethanol sedation (Fig. $4 D$; data not shown). Together, these data suggest that DopEcR is required in particular subsets of neurons, including cholinergic and peptidergic neurons, to promote ethanol-induced sedation.

\section{EGFR knockdown alleviates the sedation-resistant phenotype in DopEcR mutants}

Next, we sought to understand the downstream mechanisms by which DopEcR influences ethanol-induced sedation. Previous studies have demonstrated that EGF signaling plays a bidirectional role in ethanol-induced sedation, with activation of EGFR/ ERK signaling increasing resistance but its suppression decreasing resistance to ethanol (Corl et al., 2009; Eddison et al., 2011). Since DopEcR has also been reported to activate the MAPK/ERK cascade in heterologous cell culture systems (Srivastava et al., 2005), we reasoned that DopEcR might interact with the EGFR/ERK pathway to modulate ethanol-induced sedation. We tested this hypothesis by examining sedation in the context of suppressed EGFR signaling in a DopEcR mutant background. As reported previously (Corl et al., 2009), when Egfr-RNAi was expressed in all neurons with elav-GAL4, ethanol-induced sedation was hastened compared with control flies (data not shown). Panneuronal expression of $E g f r-R N A i$ also led to more rapid ethanol sedation in DopEcR ${ }^{P B 1}$ mutants (Fig. 5A). Similarly, expression of Egfr-RNAi in DopEcR ${ }^{G A L 4}$ mutants by its intrinsic GAL4 led to even more rapid sedation than that of Egfr-RNAi controls (Fig. 
A

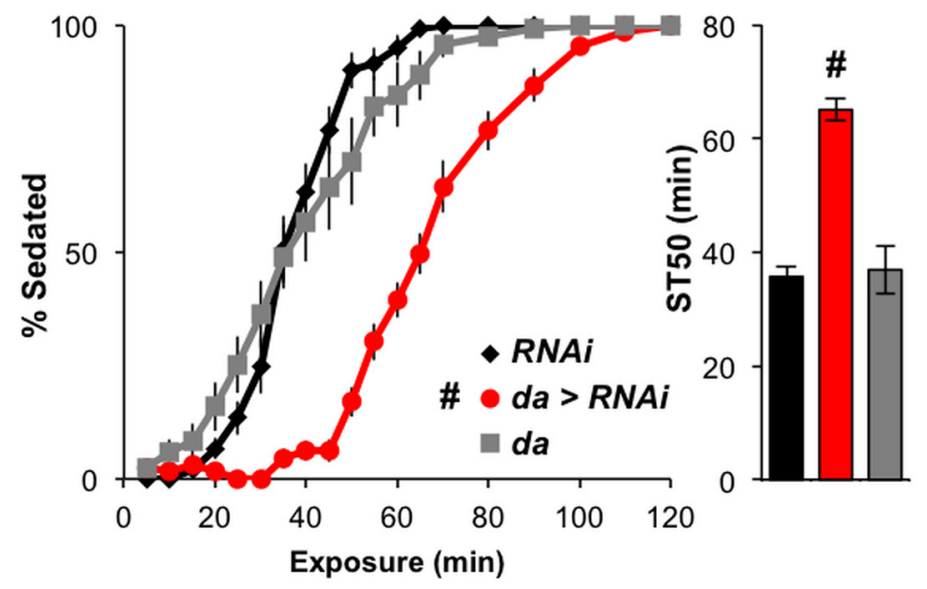

B

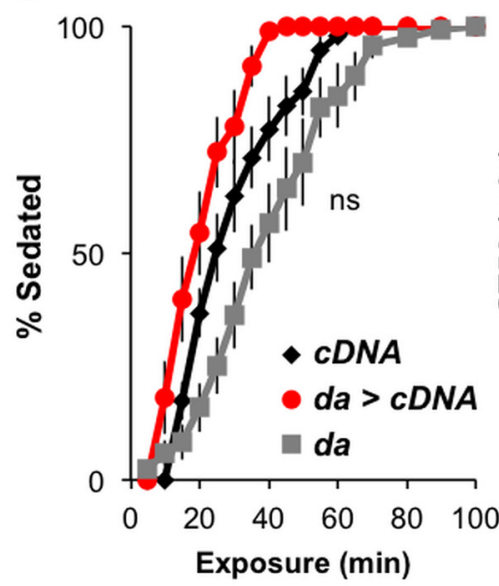

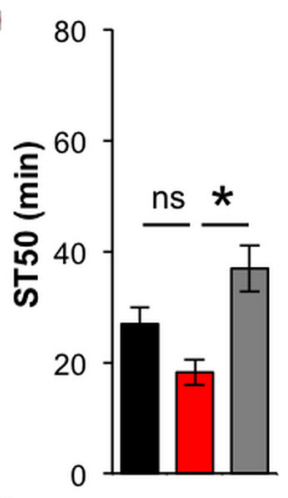

C

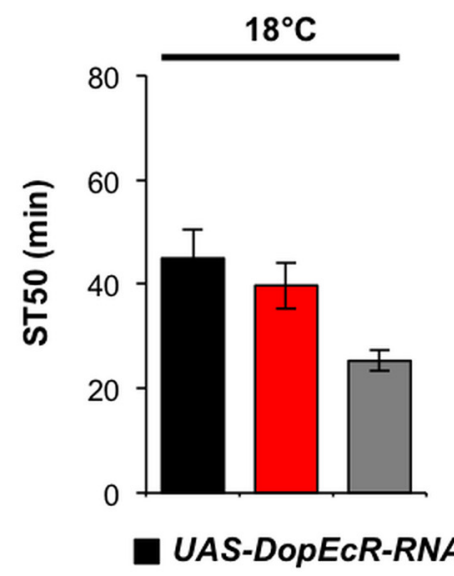

D

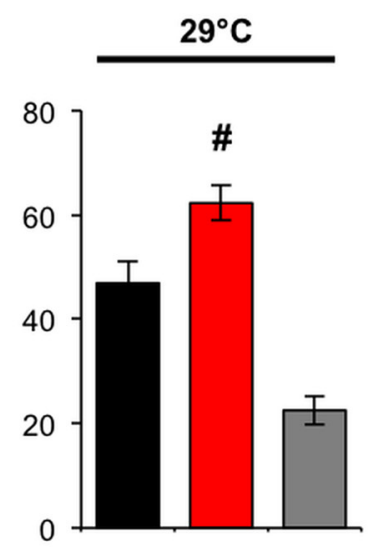

E

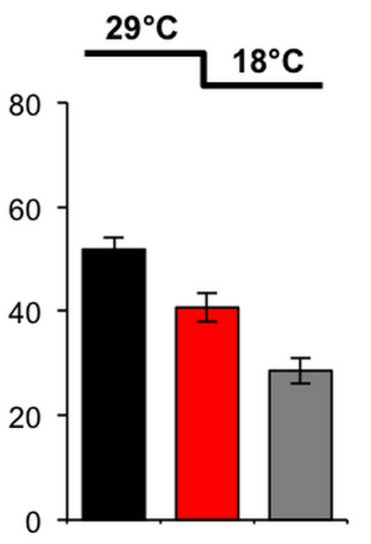

$\mathbf{F}$

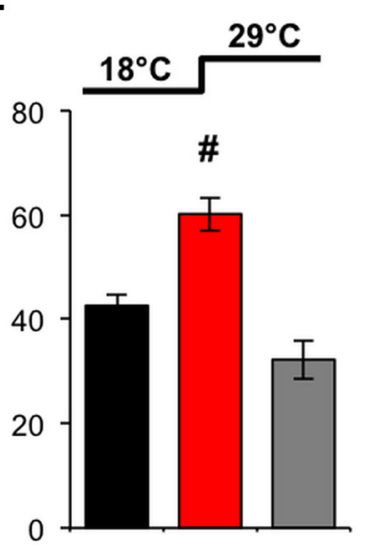

Figure 3. Normal ethanol-induced sedation requires DopEcR expression in the adult. $\boldsymbol{A}, \boldsymbol{B}$, Ethanol-induced sedation in the presence and absence of a ubiquitously expressed $D$ op $E C R$ - $R N A i(\boldsymbol{A})$ or DopEcR cDNA (B). Left, Percentage of flies exhibiting sedation. Right, ST50. da $>R N A i$ (UAS-DopECR-RNAi/ $+;$ da-GAL4/+, $n=13$ ) significantly increased resistance to ethanol sedation compared with RNAi (UAS-DopECR-RNAi/+, $n=14)$ and da-GAL4 (da-GAL4/+, $n=12$ ) controls $(\boldsymbol{A})$. da $>C D N A$ (da-GAL4/UAS-DopECR-CDNA, $n=9)$ showed a trend to increase sensitivity to become sedated compared with CDNA (UAS-DopECR-CDNA/,$+ n=10)$ and da-GAL4 (da-GAL4/+, $n=12$ ) controls $(\boldsymbol{B}) . \boldsymbol{C}-\boldsymbol{F}$, Effects of temporal knockdown of DopEcR expression on ethanol-induced sedation. Flies were either reared at constant temperature $\left(\boldsymbol{C}, 18^{\circ} \mathrm{C} ; \boldsymbol{D}, 29^{\circ} \mathrm{C}\right)$ or transferred to a different temperature after eclosion $\left(\boldsymbol{E}, 29^{\circ} \mathrm{C} \rightarrow 18^{\circ} \mathrm{C} ; \boldsymbol{F}, 18^{\circ} \mathrm{C} \rightarrow 29^{\circ} \mathrm{C}\right)$. Adult-specific knockdown of $D$ opE $C R$ expression resulted in increased resistance to ethanol-induced sedation compared with RNAi and da-GAL4 controls; UAS-DopECR-RNAi/+;da-GAL4/tub-GAL80 $0^{\text {ts }}(n=11,12,11,3$ for $\mathbf{C}-\boldsymbol{F}$ ), UAS-DopEcR-RNAi/+;tub-GAL80 $0^{\text {ts }} /+(n=9,10,12,8)$, and da-GAL4/+ $(n=8,7,6,6)$. All figures display averages with SEM, with two-way repeated-measures ANOVA on ranks, Holm-Sidak $(\boldsymbol{A}$ and $\boldsymbol{B}$, left) and Mann-Whitney rank sum tests $(\boldsymbol{A}$ and $\boldsymbol{B}$, right; $\boldsymbol{C}-\boldsymbol{F})$. \#p $<0.05$ from RNAi and $d a-G A L 4$ controls and * $p<0.05$ from da-GAL4 control.

$5 B)$. These behavioral observations for DopEcR mutants implied that their resistance to ethanol-induced sedation is the result of enhanced EGFR signaling in neurons. The strength of EGFR signaling is often estimated based on the degree of phosphorylation of the downstream effector ERK. Western blot analyses revealed that, although total ERK levels were lower in the heads of DopEcR mutants, the total levels of p-ERK were significantly increased relative to those of control flies (Fig. $5 C$ ). This result suggests that in DopEcR mutants the EGFR/ERK pathway is aberrantly activated and contributes to their increased resistance to ethanolinduced sedation.

\section{Dopaminergic mutants $D A T^{f m n}$ and $D o p R^{P L 00420}$ display} normal ethanol sedation

Since both dopamine and ecdysone are known ligands for DopEcR (Srivastava et al., 2005), we examined whether either was important for the effects of DopEcR in ethanol-induced sedation. Dopamine mediates ethanol-induced enhancement of locomotor activity in Drosophila (Bainton et al., 2000; Kong et al., 2010), as well as in mammals (Shen et al., 1995; Boileau et al., 2003;
Budygin et al., 2003). However, the manipulation of dopaminergic neurotransmission, by either genetic or pharmacologic means, does not appear to influence ethanol-induced sedation in flies (Bainton et al., 2000; Kong et al., 2010). Consistent with these findings, we observed that ethanol-induced sedation was not affected by either an increase in synaptic dopamine signaling in flies defective for dopamine transport $\left(D A T^{f m n}\right)$, or a reduction in dopamine signaling in flies expressing hypomorphic dopamine D1-like receptors (Dop $R^{P L O 0420}$; Fig. 6, red). Double mutants for each of these mutations with DopEcR ${ }^{G A L 4}$ were also created and examined for potential genetic interactions. The sedation phenotype of the DAT ${ }^{f m n} ; D o p E c R^{G A L 4}$ mutants was indistinguishable from that of DopEcR ${ }^{G A L 4}$ mutants. The ethanolinduced sedation of DopR ${ }^{P L 00420} D o p E c R^{G A L 4}$ double mutants was similarly, but slightly less, resistant than the DopEcR ${ }^{G A L 4}$ mutant (Fig. 6, green). These results together with previous findings (Bainton et al., 2000; Kong et al., 2010) indicate that dopamine signaling does not significantly contribute to DopEcR-dependent regulation of ethanol-induced sedation. 
A

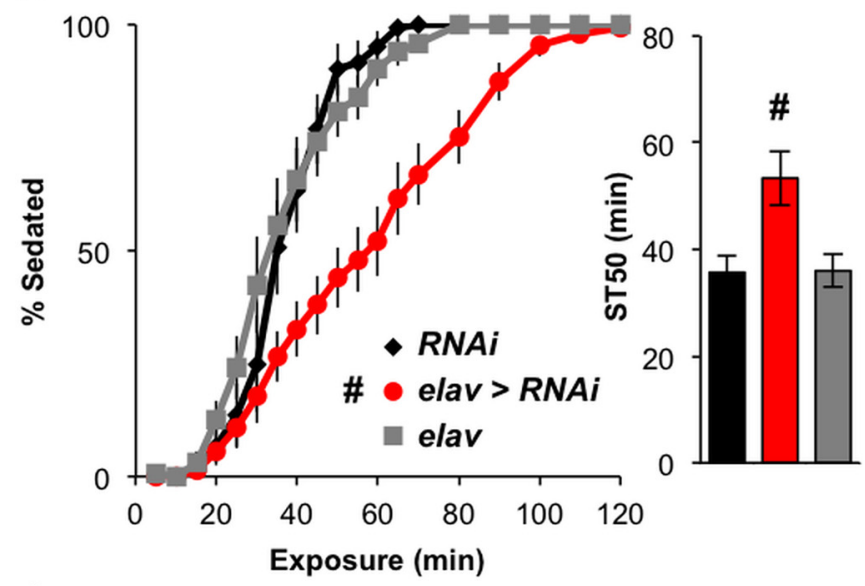

C

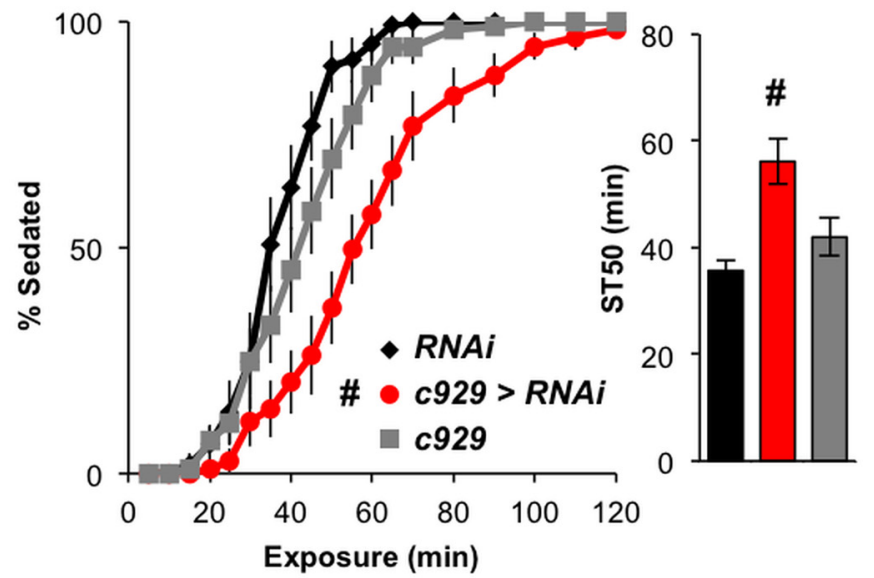

B

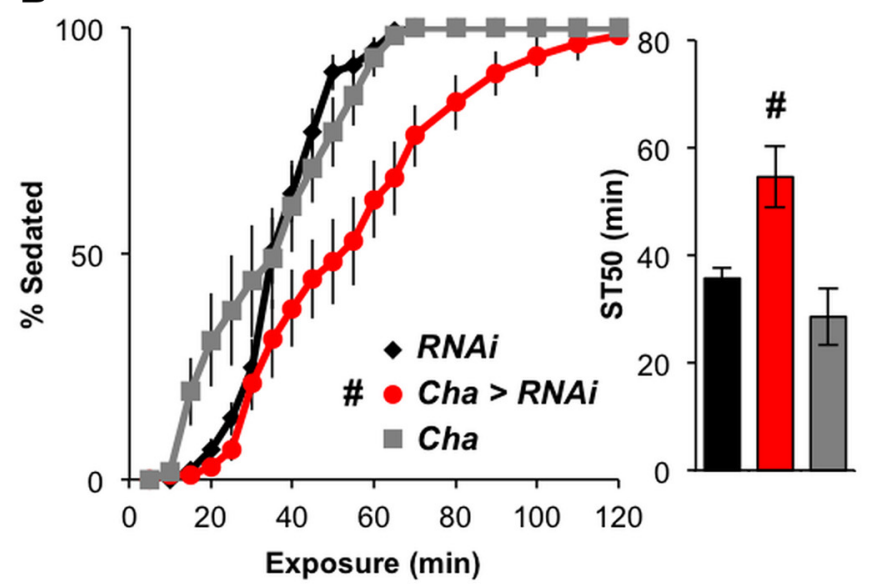

D

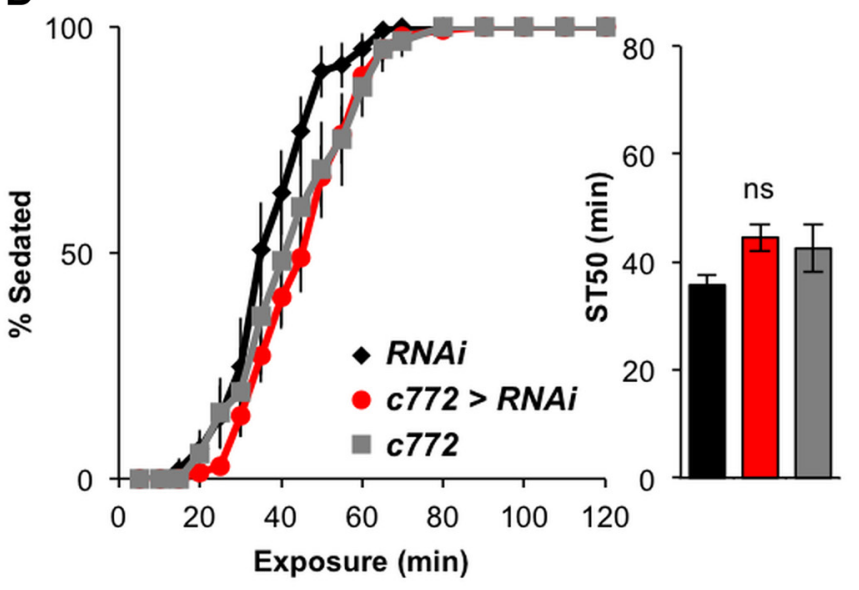

Figure 4. Resistance to ethanol-induced sedation is promoted by DopECR-RNAi expression in cholinergic or peptidergic neurons. $\boldsymbol{A}-\boldsymbol{D}$, Effects of neuronal expression of DopEcR-RNAi on ethanol-induced sedation. Left, Percentage of flies exhibiting sedation. Right, ST50.DopEcR knockdown in all ( $\boldsymbol{A})$, cholinergic $(\boldsymbol{B})$, or peptidergic neurons (C), but not in mushroom body neurons ( $\boldsymbol{D})$, significantly increased resistance to ethanol sedation compared with controls; elav $>R N A i($ elav-GAL4/Y;;UAS-DopEcR-RNAi/,$+ n=15), C h a>R N A i(C h a-G A L 4 /+; U A S-D o p E C R-R N A i /+, n=11)$, c929>RNAi (c929-GAL4/+;UAS-DopECR-RNAi/+, $n=11), c 772>$ RNAi (c772-GAL4/+;UAS-DopEcR-RNAi/+, $n=14)$, RNAi (UAS-DopECR-RNAi/+, $n=14)$, elav (elav-GAL4/+, $n=13)$, Cha (Cha-GAL4/+, $n=6), c 929$ (c929-GAL4/+, $n=11)$, and c772 (c772-GAL4/+, $n=6$ ). All figures display averages with SEM, with two-way repeated-measures ANOVA on ranks, Holm-Sidak (left), and Mann-Whitney rank-sum tests (right). \#p $<0.05$ from both RNAi and GAL4 controls.

\section{Ecdysone negatively modulates DopEcR during} ethanol exposure

To investigate the potential role of ecdysone in ethanol sedation, we first fed control $\left(w^{1118}\right)$ flies the active form of ecdysone, 20E. Acute supplementation for $24 \mathrm{~h}$ before ethanol exposure did not significantly change ethanol-induced sedation (ST50: vehicle, $43 \pm 5 \mathrm{~min}, n=7 ; 20 \mathrm{E}, 47 \pm 4 \mathrm{~min}, n=$ $7)$. We then used the ecdysone-defective mutant Dominant temperature-sensitive-3 (DTS-3; Holden and Suzuki, 1973) to modulate endogenous ecdysone levels. The DTS-3 mutation is homozygous lethal regardless of temperature, but heterozygous mutants survive at $22^{\circ} \mathrm{C}$ (permissive temperature) and only fail to molt at $29^{\circ} \mathrm{C}$ (restrictive temperature) due to reduced levels of ecdysone (Holden and Suzuki, 1973). The ethanol sensitivity of control and DTS-3 (DTS-3/+) flies reared continuously at $18^{\circ} \mathrm{C}$ did not differ significantly (ST50: $32 \pm 4$ $\min , n=6 ; 25 \pm 2 \min , n=12$, respectively). However, when reared at $18^{\circ} \mathrm{C}$ during development and transferred to $29^{\circ} \mathrm{C}$ after eclosion, DTS-3 flies were sedated more quickly than controls upon ethanol exposure (Fig. 7A). This suggests that reducing ecdysone levels in the adult promotes ethanolinduced sedation, which is the opposite of the DopEcR loss-of- function phenotype. To examine the interaction between DTS-3 and DopEcR, we generated flies that were heterozygous for DTS-3 and homozygous for DopEcR $R^{G A L 4}$. When reared at $18^{\circ} \mathrm{C}$ and transferred to $29^{\circ} \mathrm{C}$ after eclosion, these double mutants showed significant resistance to ethanol-induced sedation (comparable to that of DopEcR ${ }^{G A L 4}$ mutants; Fig. $7 A$ ). This finding suggests that DopEcR function is required for the sensitizing effect of DTS-3 on ethanol sedation. In further support of this, ubiquitous expression of DopEcR-RNAi in DTS-3/+ mutant flies also mitigated the sensitivity of ecdysone-defective flies to sedation (Fig. 7B). Together these results suggest that reducing posteclosion ecdysone levels results in DopEcR-dependent promotion of ethanol-induced sedation.

To test the potential negative relationship between ecdysone and DopEcR in controlling ethanol-induced sedation, we again looked at the effects of acute feeding of $20 \mathrm{E}$. The decreased sedation resistance of DTS-3 mutants shifted to the restrictive temperature after eclosion was slightly mitigated by treatment with 20E, but statistical significance was not reached (DTS-3 ST50: vehicle, $23 \pm 3 \mathrm{~min}, n=11$; 20E, $29 \pm$ $3 \mathrm{~min}, n=12$ ). In contrast, the decreased sedation resistance 
A

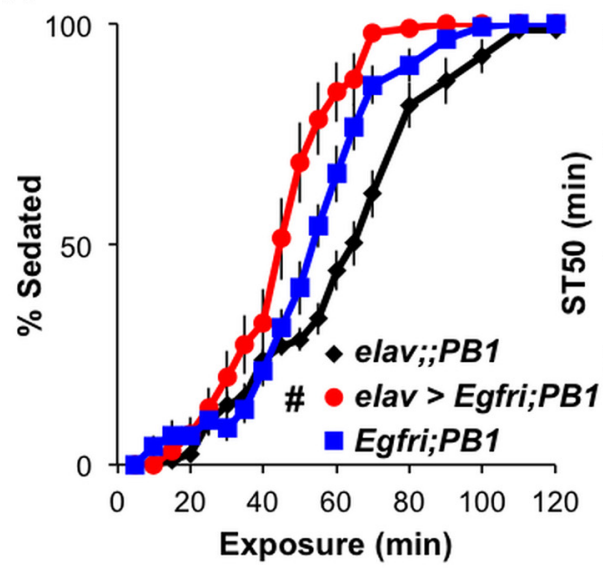

B

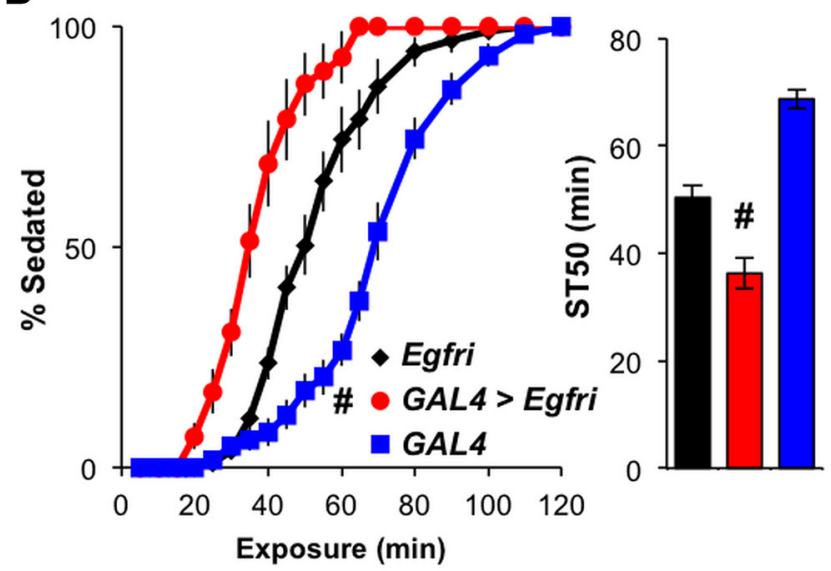

C
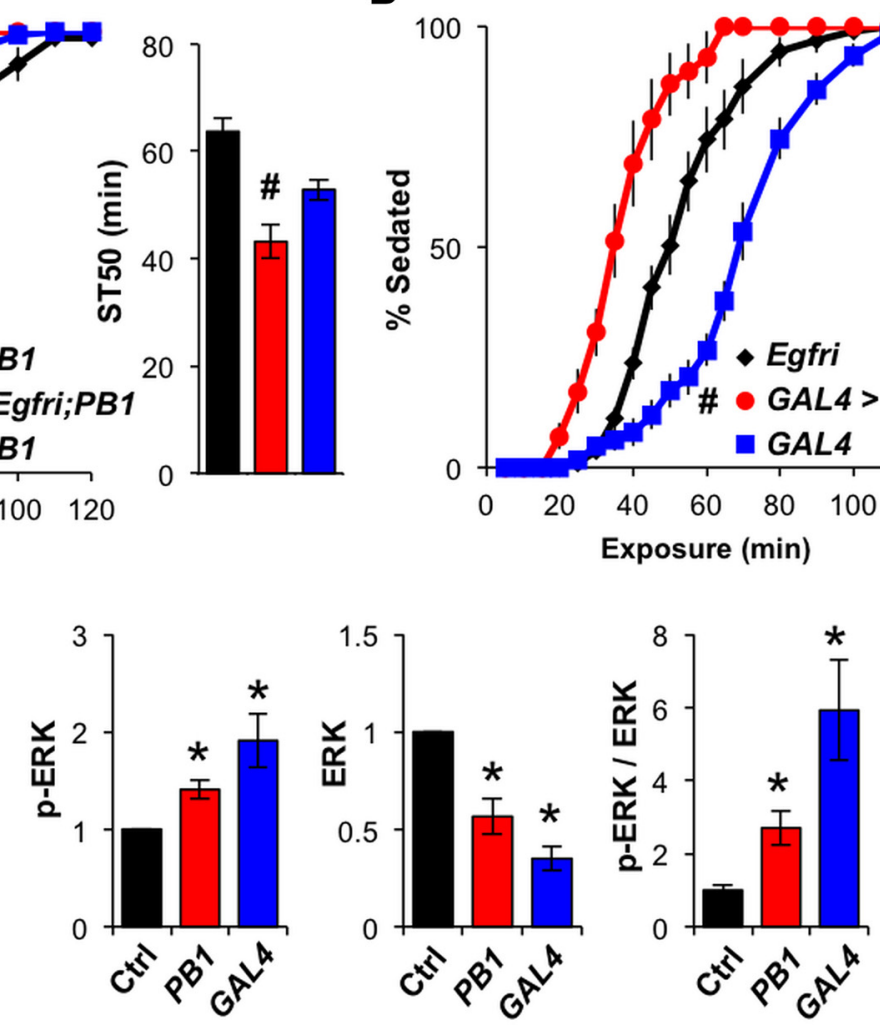

Figure 5. Inhibition of downstream EGFR/ERK signaling mitigates the DopECR mutant phenotype in ethanol-induced sedation. $A$, Pan-neuronal expression of Egfr-RNAi in DopECR ${ }^{P B 1}$ mutants. Left, Percentage of flies exhibiting sedation. Right, ST50; elav >Egfri; PB1 (elav-GAL4/Y;UAS-Egfr-RNAi/+;DopEcCPB1, $n=10)$, elav;; PB1 (elav-GAL4/Y;;DopECR $R^{P B 1}, n=13$ ), and Egfri;PB1 (UAS-Egfr-RNAi/+;DopEc $\left.R^{P B 1}, n=9\right)$ ). B, Expression of the Egfr-RNAi in DopECR ${ }^{G A L 4}$ mutants using its GAL4 activity; GAL4> Egfri (UAS-Egfr-RNAi;DopEcR $\left.{ }^{G A L 4}, n=10\right), R N A i$ (UAS-Egfr-RNAi, $n=8$ ), and GAL4 (DopECR $\left.{ }^{G A L 4}, n=13\right)$. Knockdown of EGFR expression in DopEC $R^{P B 1}(\boldsymbol{A})$ or DopEC $R^{G A L 4}(\boldsymbol{B})$ mutants significantly increased their sensitivity to ethanol-induced sedation. $C$, Left, Representative Western blots for basal p-ERK, total ERK, and $\alpha$-tubulin in head lysates of Ctrl $\left(w^{1118}\right), P B 1\left(D o p E C R^{P B 1}\right)$, and GAL4 (DopECR $\left.{ }^{G A L 4}\right)$ flies. Right, Densitometric quantification of the bands $(n=4)$. All figures display averages with SEM, with two-way repeated-measures ANOVA on ranks, Holm-Sidak $(\boldsymbol{A}$ and $\boldsymbol{B}$, left), Mann-Whitney rank-sum tests ( $\boldsymbol{A}$ and $\boldsymbol{B}$, right), and ANOVA on ranks, Dunn's versus control (C). $\# p<0.05$ from both RNAi and GAL4 controls and ${ }^{*} p<0.05$ from the wild-type control.
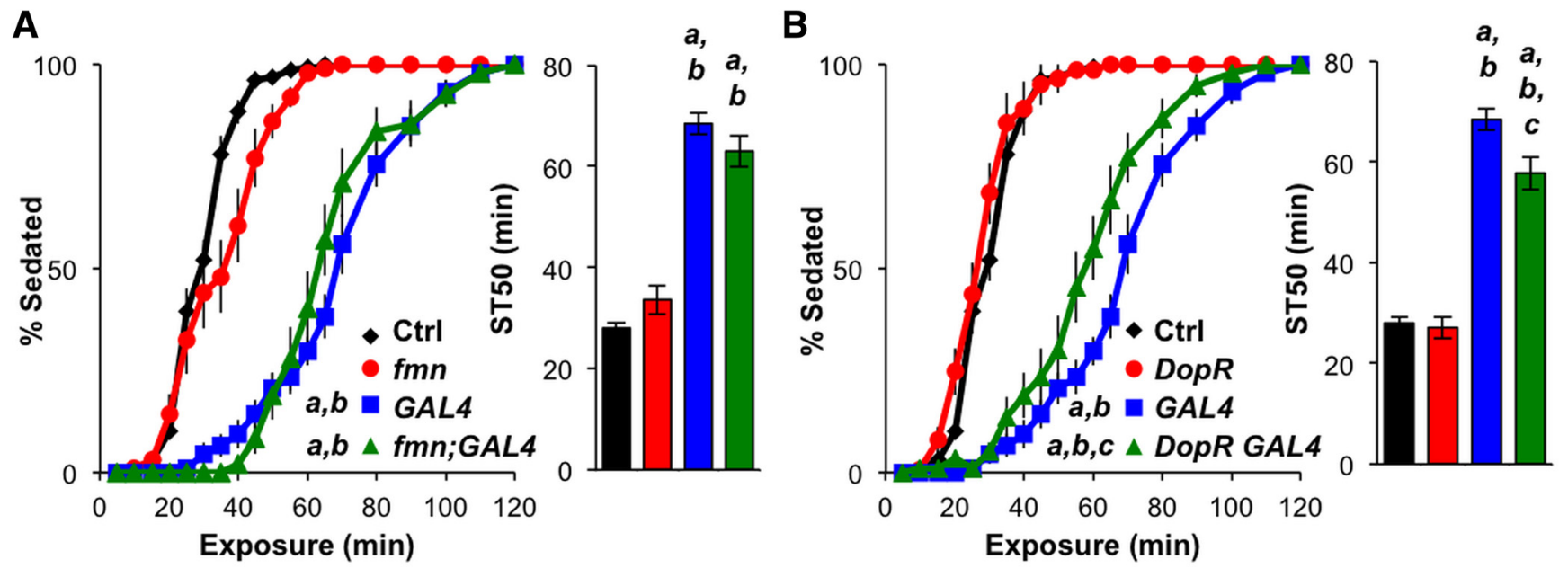

Figure 6. Ethanol-induced sedation is normal in dopaminergic mutants $D A T^{f m n}$ and DopR ${ }^{P L 00420} . \boldsymbol{A}, \boldsymbol{B}$, Effects of DAT and DopR mutations on ethanol-induced sedation in the presence or absence of $D o p E c R^{G A L 4}$. Left, Percentage of flies exhibiting sedation. Right, ST50. Both DAT and DopR mutations $\left(A, D A T^{f m n} ; B, D o p R^{P L 00420}\right)$ did not have a significant effect on ethanol-induced sedation regardless of $D$ opEC $R$

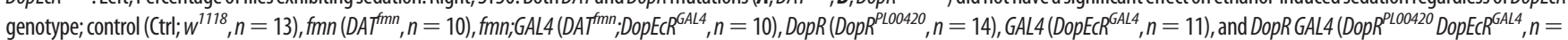
10). Two-way repeated-measures ANOVA on ranks, Holm-Sidak (left), and ANOVA on ranks, Dunn's multiple comparisons (right). a-crepresent $p<0.05$ from first, second, third genotype displayed.

of flies ubiquitously overexpressing $D o p E c R$ was significantly reversed after feeding with 20E (Fig. 7C). Collectively, these findings suggest that ecdysone negatively regulates DopEcR activity to ultimately control the timing of ethanol-induced sedation.

\section{Discussion}

In humans, resistance to ethanol-induced sedation positively correlates with an individual's risk for becoming an alcoholic (Schuckit et al., 2000). Therefore, a better understanding of the 
A

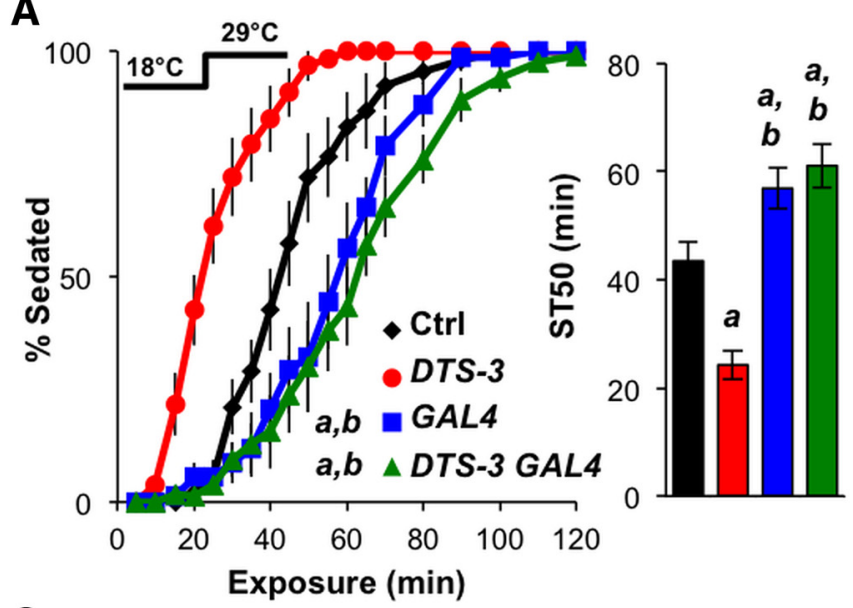

C

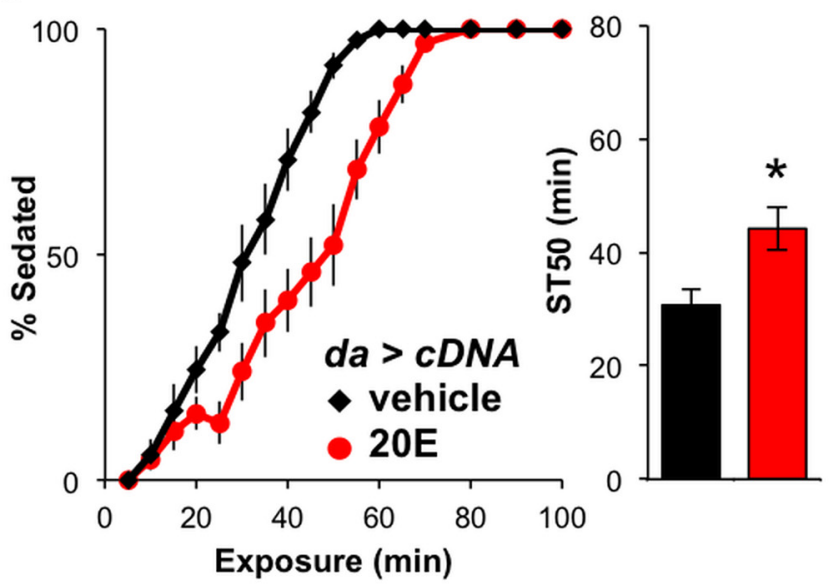

B

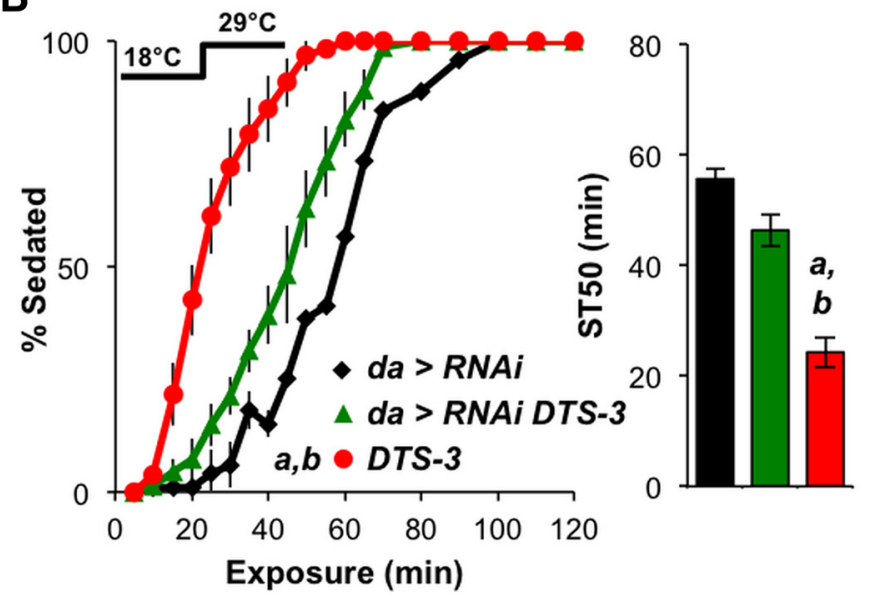

Figure 7. The active form of ecdysone, 20E, suppresses the sedation-promoting activity of DopEcR upon ethanol exposure. $\boldsymbol{A}, \boldsymbol{B}$, Effects of $D T S$-3 on ethanol-induced sedation. Left, Percentage of flies exhibiting sedation. Right, ST50. The temperature-sensitive DTS-3 mutants were raised at $18^{\circ} \mathrm{C}$ then transferred to $29^{\circ} \mathrm{C}$ after eclosion. As compared with control (Ctrl), DTS-3 flies showed decreased resistance to ethanol sedation $(\boldsymbol{A})$. Reduction of DopEcR function by means of DopEcR ${ }^{G A L 4}(\boldsymbol{A})$ or DopECR knockdown $(\boldsymbol{B})$ eliminated the effect of DTS-3 on ethanol sedation; $C \operatorname{trl}\left(w^{1118} / Y, n=9\right), D T S-3\left(w^{1118} / Y ; D T S-3 /+, n=12\right)$, GAL4 ( $\left.w^{1118} / Y ; D o p E C R^{G A L 4}, n=7\right), D T S-3$ GAL4 ( $w^{1118} / Y ; D T S-3 /+$ DopEc $\left.R^{G A L 4}, n=8\right), d a>R N A i$ (UAS-DopEcR-RNAi/+;da-GAL4/+, $n=10$ ) and da>RNAi DTS-3 (UAS-DopECR-RNAi/+;da-GAL4/DTS-3, $n=8$ ). C, Effects of 20E feeding on ethanol-induced sedation in flies ubiquitously overexpressing DopECR-CDNA. Left, Percentage of flies exhibiting sedation. Right, ST50. Resistance to ethanol sedation was increased in da $>C D N A$ (UAS-DopEcR-CDNA/daGAL4) flies fed $0.1 \mathrm{~mm} \mathrm{20E}(n=10)$ in food for $24 \mathrm{~h}$ before ethanol exposure compared with those fed vehicle $(n=9)$. All figures display averages with SEM, with two-way repeated-measures ANOVA on ranks, Holm-Sidak ( $\boldsymbol{A}$ and $\boldsymbol{B}$, left), and ANOVA on ranks, Dunn's multiple comparisons ( $\boldsymbol{A}$ and $\boldsymbol{B}$, right), and Mann-Whitney rank-sum test ( $\boldsymbol{C}$, right). a and b represent $p<0.05$ from first and second genotype displayed; * $p<0.05$ from the vehicle control.

biological mechanisms underlying ethanol-induced sedation is of great clinical importance. Fruit flies have proven to be a useful model in which to study evolutionarily conserved genes involved in drug addiction (Kaun et al., 2012). In the study reported here, we found that mutants for Drosophila DopEcR, a unique GPCR with dual specificity for dopamine and ecdysone, exhibited resistance to ethanol-induced sedation despite having normal initial behavioral responses (i.e., loss of postural control) as well as proper ethanol absorption and metabolism (Fig. 2). These findings indicate that DopEcR is necessary for normal sedation during ethanol exposure. RNAi-mediated knockdown of DopEcR, either after eclosion (Fig. 3) or in specific neuronal populations, including peptidergic and cholinergic neurons (Fig. 4), recapitulated the sedation-resistant phenotype of DopEcR mutants. Our study further implicates EGFR/ERK signaling downstream of DopEcR in this process (Fig. 5), and suggests that ecdysone may negatively regulate DopEcR to influence ethanol-induced sedation (Fig. 7). This and future investigations into the actions of DopEcR offer important insight into the elusive function and mechanism of GPCR-mediated noncanonical steroid signal- ing, particularly in the context of the physiological response to alcohol.

\section{Role of DopEcR ligands in ethanol-induced sedation}

Dopamine and ecdysone are known ligands of DopEcR in flies (Srivastava et al., 2005; Inagaki et al., 2012; Ishimoto et al., 2013). As shown by others (Bainton et al., 2000; Kong et al., 2010) and in our study (Fig. 6), dopaminergic signaling is not significantly involved in the sedating effect of ethanol in Drosophila. Thus, dopamine is not relevant to the activation of DopEcR to promote ethanol-induced sedation. Interestingly, however, DopEcRmediated dopaminergic signaling may play a role in different aspects of the behavioral response to ethanol, namely ethanolinduced hyperactivity, which depends on conventional dopamine signaling via the D1-like receptor, DopR (Kong et al., 2010). Our recent experiments show that this dopamine-dependent behavioral response to ethanol is exaggerated in DopEcR mutants (data not shown). This observation raises the possibility that, although dopamine is not involved in the DopEcR-mediated regulation of ethanol-induced sedation, it may act through 
DopEcR to counteract DopR-mediated ethanol-induced hyperactivity.

In the case of ecdysone, we found that acute $20 \mathrm{E}$ feeding had no effect on the ethanol-induced sedation of control flies. However, DTS-3 mutants, which were deficient for ecdysone synthesis in adults, displayed increased sensitivity to ethanol-induced sedation in a DopEcR-dependent manner. In addition, acute 20E feeding to flies overexpressing $D o p E c R$ led to a significant increase in their resistance to ethanol-induced sedation (Fig. 7). These data suggest that ecdysone can negatively affect DopEcR activity that promotes ethanol-induced sedation.

Despite the resistance of $D o p E c R$ mutants to ethanol-induced sedation, our results indicate that neither dopamine nor ecdysone is required for the activation of DopEcR during ethanol exposure to promote sedation. One potential explanation for this is that other unidentified DopEcR agonists are responsible for promoting ethanol-induced sedation. Another possibility is that DopEcR exerts agonist-independent activity. Historically, GPCRs were thought to have two distinct conformations - an active and an inactive statecontingent upon ligand availability. It is now accepted that GPCRs occupy a wide range of conformational states that influence the extent and specificity of downstream signaling events. Also, in addition to the effects of agonists and inverse agonists, which stabilize unique GPCR states (Rasmussen et al., 2011), many GPCRs inherently show agonist-independent basal activity (Bond and Ijzerman, 2006; Nakashima et al., 2013). Considering our experimental findings, it is possible that DopEcR exerts agonist-independent activity to promote ethanol-induced sedation, and that this activity can be suppressed by ecdysteroids acting as inverse agonists. This hypothesis would explain how reduced ecdysone levels in DTS-3 mutants could lead to DopEcR disinhibition, and thus decreased resistance to ethanol-induced sedation, a result similar to that of overexpressing $D o p E c R$. Although this scenario predicts that increasing levels of $20 \mathrm{E}$ will increase ethanol-induced resistance to sedation, an effect of acute $20 \mathrm{E}$ feeding was only observed in flies overexpressing $D o p E c R$. There are several potential reasons for this discrepancy. First, perhaps the majority of DopEcR receptors are already occupied by $20 \mathrm{E}$, or other endogenous steroidal ligands for DopEcR, such as makisterone A and the prohormone ecdysone. Second, it is possible that ecdysteroids, like neurohormones, have refined site-specific actions and $20 \mathrm{E}$ feeding fails to recapitulate localized ecdysone signaling necessary for DopEcR-mediated processes. Third, genomic and nongenomic actions of the canonical ecdysone receptor EcR may affect DopEcR-mediated ecdysone signaling in controlling the response to alcohol. It is also important to consider that the availability, specificity, and competition of GPCR ligands is often a complex and site-specific phenomenon (Kenakin and Christopoulos, 2013). The extent of DopEcR expression may even affect the neuroactive pools of 20E, which then modulates nuclear ecdysteroid (EcR) signaling. Further molecular and cellular analyses are required to test the validity of these possibilities and fully understand the mechanisms by which $20 \mathrm{E}$ regulates behavioral response to ethanol.

\section{EGFR/ERK signaling mediates DopEcR-dependent behavioral response to ethanol}

Our finding that DopEcR negatively affects EGFR/ERK signaling to promote ethanol sedation (Fig. 5) is consistent with prior investigations, in both flies and rodents, demonstrating that the EGFR/ERK activity positively correlates with resistance to ethanol-induced sedation (Corl et al., 2009). Previous studies have also provided insight into the spatial requirements of EGFR/ERK signaling in ethanol-induced sedation. Specifically, reducing EGFR/ERK in either insulin-producing (dilp2+) or do- paminergic $(\mathrm{TH}+)$ neurons increases resistance to ethanolinduced sedation (Corl et al., 2009). At first glance our data seem incompatible with these models since restricted DopEcR-RNAi expression in $\mathrm{TH}+$ or dilp $2+$ neurons did not significantly affect ethanol-induced sedation (data not shown). In mammals, however, EGFR can be transactivated or transinhibited by alcohol itself or GPCRs, via intracellular or intercellular mechanisms (Mill et al., 2009; Berasain et al., 2011). Therefore, there is the possibility that DopEcR may modulate EGFR signaling, via intercellular mechanisms, in neurons that do not express DopEcR. It is also possible that DopEcR independently influences the EGFR and ERK pathways. Indeed, DopEcR-mediated ERK phosphorylation was previously observed in Chinese hamster ovary cells, which are known to lack EGFR expression (Srivastava et al., 2005). Altogether, our data show that DopEcR may influence EGFR/ERK signaling either directly or indirectly to alter the timing of ethanol-induced sedation.

Unlike the sedation-resistant happyhour (Ste20 kinase) mutants, which showed increased p-ERK levels only after short exposure to ethanol vapor (Corl et al., 2009), DopEcR mutants exhibited higher basal levels of p-ERK (Fig. 5). We suspect that this disparity is caused by distinct roles of DopEcR and Ste20 kinase in regulation of EGFR/ERK signaling; DopEcR may be involved in maintaining the steady-state activity of EGFR signaling, whereas Ste20 kinase may play a role in modulating the acute response of the signaling pathway upon exposure to ethanol. We also found that EGFR/ERK signaling and DopEcR function have different temporal requirements. Experiments using panneuronal knockdown of EGFR indicate that the EGFR/ERK pathway is required both during development and in adulthood to influence ethanol-induced sedation sensitivity (Eddison et al., 2011). Our findings using DopEcR-RNAi in combination with the TARGET system suggest that DopEcR function is required, particularly after eclosion, for normal sensitivity to the sedative effect of ethanol. We cannot rule out the possibility that DopEcR plays a role in posteclosion development, such as neuronal remodeling. However, the results shown in Figures 3-5 support the idea that DopEcR is involved in adult neuronal processing, including the modulation of EGFR/ERK signaling, which is critical for the proper sedative response to ethanol.

\section{Functional similarities of DopEcR to vertebrate GPER1}

DopEcR is similar to GPER1, the vertebrate GPCR for $17 \beta$-estradiol, in terms of its cellular location, signaling properties, and pharmacology (Evans et al., 2014). In particular, GPER1 shares downstream effectors with DopEcR, modulating EGFR/ERK and cAMP signaling pathways (Olde and Leeb-Lundberg, 2009). In addition to the involvement of EGFR/ERK signaling discussed above, we have evidence to suggest that DopEcR requires cAMP signaling to control ethanol sedation timing. Specifically, a cAMP phosphodiesterase mutation $(\text { dunce })^{1}$ ), but not by an adenylyl cyclase mutation (ruta$b a g a^{I}$ ), eliminated the sedation resistance of DopEcR mutants (data not shown). These findings are consistent with those of previous work demonstrating that cAMP is crucial for the conserved behavioral response to alcohol and that cAMP signaling can be activated downstream of DopEcR (Moore et al., 1998; Srivastava et al., 2005; Ishimoto et al., 2013).

GPER1 also responds to dopamine in a dose-dependent manner when expressed in Xenopus oocytes (Evans et al., 2014). Furthermore, both the DopEcR and GPER1 protein sequences contain putative cholesterol recognition/interaction amino acid consensus sequences that have been proposed as a sterol-binding domain in GPCRs (Jafurulla et al., 2011). In consideration of 
these intriguing parallels and our current findings, the potential function of GPER1 in the response to ethanol warrants further investigation. The results obtained from our DopEcR experiments emphasize the importance of a unique steroid GPCR in alcohol response and lay the foundation for future works to probe the evolutionarily conserved mechanisms underlying the roles of G-protein-coupled steroid receptors in AUDs.

\section{Notes}

Supplemental material for this article is available at https://youtu.be/ K9yrRXbhitg. DopEcR mutants are resistant to ethanol-induced sedation. This video of control ( $\left.w^{1118}\right)$ flies (left) and DopEcR $R^{G A L 4}$ mutants (right) after $30 \mathrm{~min}$ of ethanol exposure demonstrates the robust sedation resistance of $D o p E c R$ mutants. The frame rate has been sped up $3 \times$ real time. This material has not been peer reviewed.

\section{References}

Abrieux A, Debernard S, Maria A, Gaertner C, Anton S, Gadenne C, Duportets L (2013) Involvement of the G-protein-coupled dopamine/ecdysteroid receptor DopEcR in the behavioral response to sex pheromone in an insect. PLoS One 8:e72785. CrossRef Medline

Abrieux A, Duportets L, Debernard S, Gadenne C, Anton S (2014) The GPCR membrane receptor, DopEcR, mediates the actions of both dopamine and ecdysone to control sex pheromone perception in an insect. Front Behav Neurosci 8:312. CrossRef Medline

Bainton RJ, Tsai LT, Singh CM, Moore MS, Neckameyer WS, Heberlein U (2000) Dopamine modulates acute responses to cocaine, nicotine and ethanol in Drosophila. Curr Biol 10:187-194. CrossRef Medline

Berasain C, Ujue Latasa M, Urtasun R, Goni S, Elizalde M, Garcia-Irigoyen O, Azcona M, Prieto J, Avila MA (2011) Epidermal growth factor receptor (EGFR) crosstalks in liver cancer. Cancers (Basel) 3:2444-2461. CrossRef Medline

Boileau I, Assaad JM, Pihl RO, Benkelfat C, Leyton M, Diksic M, Tremblay RE, Dagher A (2003) Alcohol promotes dopamine release in the human nucleus accumbens. Synapse 49:226-231. CrossRef Medline

Bond RA, Ijzerman AP (2006) Recent developments in constitutive receptor activity and inverse agonism, and their potential for GPCR drug discovery. Trends Pharmacol Sci 27:92-96. CrossRef Medline

Brand AH, Perrimon N (1993) Targeted gene expression as a means of altering cell fates and generating dominant phenotypes. Development 118:401-415. Medline

Budygin EA, John CE, Mateo Y, Daunais JB, Friedman DP, Grant KA, Jones SR (2003) Chronic ethanol exposure alters presynaptic dopamine function in the striatum of monkeys: a preliminary study. Synapse 50:266-268. CrossRef Medline

Cavaliere S, Gillespie JM, Hodge JJ (2012) KCNQ channels show conserved ethanol block and function in ethanol behaviour. PLoS One 7:e50279. CrossRef Medline

Chen J, Zhang Y, Shen P (2008) A protein kinase C activity localized to neuropeptide $Y$-like neurons mediates ethanol intoxication in Drosophila melanogaster. Neuroscience 156:42-47. CrossRef Medline

Corl AB, Berger KH, Ophir-Shohat G, Gesch J, Simms JA, Bartlett SE, Heberlein U (2009) Happyhour, a Ste20 family kinase, implicates EGFR signaling in ethanolinduced behaviors. Cell 137:949-960. CrossRef Medline

Daniels RW, Rossano AJ, Macleod GT, Ganetzky B (2014) Expression of multiple transgenes from a single construct using viral $2 \mathrm{~A}$ peptides in Drosophila. PLoS One 9:e100637. CrossRef Medline

Devaud LL, Risinger FO, Selvage D (2006) Impact of the hormonal milieu on the neurobiology of alcohol dependence and withdrawal. J Gen Psychol 133:337-356. CrossRef Medline

Devineni AV, Heberlein U (2012) Acute ethanol responses in Drosophila are sexually dimorphic. Proc Natl Acad Sci U S A 109:21087-21092. CrossRef Medline

Devineni AV, Heberlein U (2013) The evolution of Drosophila melanogaster as a model for alcohol research. Annu Rev Neurosci 36:121-138. CrossRef Medline

Eddison M, Guarnieri DJ, Cheng L, Liu CH, Moffat KG, Davis G, Heberlein U (2011) arouser reveals a role for synapse number in the regulation of ethanol sensitivity. Neuron 70:979-990. CrossRef Medline

Evans PD, Bayliss A, Reale V (2014) GPCR-mediated rapid, non-genomic actions of steroids: comparisons between DmDopEcR and GPER1 (GPR30). Gen Comp Endocrinol 195:157-163. CrossRef Medline

Graveley BR, Brooks AN, Carlson JW, Duff MO, Landolin JM, Yang L, Artieri
CG, van Baren MJ, Boley N, Booth BW, Brown JB, Cherbas L, Davis CA, Dobin A, Li R, Lin W, Malone JH, Mattiuzzo NR, Miller D, Sturgill D, et al. (2011) The developmental transcriptome of Drosophila melanogaster. Nature 471:473-479. CrossRef Medline

Harlow E, Lane D (1988) Antibodies: a laboratory manual. Cold Spring Harbor, NY: Cold Spring Harbor Laboratory.

Holden JJ, Suzuki DT (1973) Temperature-sensitive mutations in Drosophila melanogaster. XII. The genetic and developmental effects of dominant lethals on chromosome 3. Genetics 73:445-458. Medline

Huang J, Zhou W, Watson AM, Jan YN, Hong Y (2008) Efficient ends-out gene targeting in Drosophila. Genetics 180:703-707. CrossRef Medline

Inagaki HK, Ben-Tabou de-Leon S, Wong AM, Jagadish S, Ishimoto H, Barnea G, Kitamoto T, Axel R, Anderson DJ (2012) Visualizing neuromodulation in vivo: TANGO-mapping of dopamine signaling reveals appetite control of sugar sensing. Cell 148:583-595. CrossRef Medline

Ishimoto H, Wang Z, Rao Y, Wu CF, Kitamoto T (2013) A novel role for ecdysone in Drosophila conditioned behavior: linking GPCR-mediated non-canonical steroid action to cAMP signaling in the adult brain. PLoS Genet 9:e1003843. CrossRef Medline

Jafurulla M, Tiwari S, Chattopadhyay A (2011) Identification of cholesterol recognition amino acid consensus (CRAC) motif in G-protein coupled receptors. Biochem Biophys Res Commun 404:569-573. CrossRef Medline

Kaun KR, Devineni AV, Heberlein U (2012) Drosophila melanogaster as a model to study drug addiction. Hum Genet 131:959-975. CrossRef Medline

Kenakin T, Christopoulos A (2013) Signalling bias in new drug discovery: detection, quantification and therapeutic impact. Nat Rev Drug Discov 12:205-216. CrossRef Medline

Kong EC, Woo K, Li H, Lebestky T, Mayer N, Sniffen MR, Heberlein U, Bainton RJ, Hirsh J, Wolf FW (2010) A pair of dopamine neurons target the D1-like dopamine receptor DopR in the central complex to promote ethanol-stimulated locomotion in Drosophila. PLoS One 5:e9954. CrossRef Medline

Koob GF (2008) A role for brain stress systems in addiction. Neuron 59: 11-34. CrossRef Medline

Lösel R, Wehling M (2003) Nongenomic actions of steroid hormones. Nat Rev Mol Cell Biol 4:46-56. CrossRef Medline

McGuire SE, Mao Z, Davis RL (2004) Spatiotemporal gene expression targeting with the TARGET and gene-switch systems in Drosophila. Sci STKE 2004:pl6. Medline

Mill CP, Chester JA, Riese DJ 2nd (2009) EGFR may couple moderate alcohol consumption to increased breast cancer risk. Breast Cancer (Dove Med Press) 1:31-38. Medline

Moore MS, DeZazzo J, Luk AY, Tully T, Singh CM, Heberlein U (1998) Ethanol intoxication in Drosophila: genetic and pharmacological evidence for regulation by the cAMP signaling pathway. Cell 93:997-1007. CrossRef Medline

Nakashima A, Takeuchi H, Imai T, Saito H, Kiyonari H, Abe T, Chen M, Weinstein LS, Yu CR, Storm DR, Nishizumi H, Sakano H (2013) Agonist-independent GPCR activity regulates anterior-posterior targeting of olfactory sensory neurons. Cell 154:1314-1325. CrossRef Medline

Olde B, Leeb-Lundberg LM (2009) GPR30/GPER1: searching for a role in estrogen physiology. Trends Endocrinol Metab 20:409-416. CrossRef Medline

Rasmussen SG, DeVree BT, Zou Y, Kruse AC, Chung KY, Kobilka TS, Thian FS, Chae PS, Pardon E, Calinski D, Mathiesen JM, Shah ST, Lyons JA, Caffrey M, Gellman SH, Steyaert J, Skiniotis G, Weis WI, Sunahara RK, Kobilka BK (2011) Crystal structure of the beta2 adrenergic receptor-Gs protein complex. Nature 477:549-555. CrossRef Medline

Schneider CA, Rasband WS, Eliceiri KW (2012) NIH Image to ImageJ: 25 years of image analysis. Nat Methods 9:671-675. CrossRef Medline

Schuckit MA, Smith TL, Landi NA (2000) The 5-year clinical course of high-functioning men with DSM-IV alcohol abuse or dependence. Am J Psychiatry 157:2028-2035. CrossRef Medline

Shen EH, Crabbe JC, Phillips TJ (1995) Dopamine antagonist effects on locomotor activity in naive and ethanol-treated FAST and SLOW selected lines of mice. Psychopharmacology (Berl) 118:28-36. CrossRef Medline

Srivastava DP, Yu EJ, Kennedy K, Chatwin H, Reale V, Hamon M, Smith T, Evans PD (2005) Rapid, nongenomic responses to ecdysteroids and catecholamines mediated by a novel Drosophila G-protein-coupled receptor. J Neurosci 25:6145-6155. CrossRef Medline

Wolf FW, Rodan AR, Tsai LT, Heberlein U (2002) High-resolution analysis of ethanol-induced locomotor stimulation in Drosophila. J Neurosci 22: 11035-11044. Medline 